\title{
A Portable Fluorescence Lifetime Spectroscopy Detector for Molecular Diagnosis ${ }^{+}$
}

\author{
Joan Canals *, Nil Franch, Oscar Alonso, Anna Vilà and Angel Diéguez \\ Department of Electronics, University of Barcelona, 08028 Barcelona, Spain; nfranch@el.ub.edu (N.F.); \\ oalonso@el.ub.edu (O.A.); avila@el.ub.edu (A.V.); adieguez@el.ub.edu (A.D.) \\ * Correspondence: jcanals@el.ub.edu \\ + Presented at the 5th International Symposium on Sensor Science (I3S 2017), Barcelona, Spain, \\ 27-29 September 2017.
}

Published: 29 November 2017

Fluorescence-based techniques are amongst the most widely used methods in molecular analysis in life science with multiple applications in clinical analysis and biomedical diagnosis. Considerable efforts have been directed toward the miniaturization of the fluorescence-based instruments, in an effort to reduce both cost and form factor for point of care (PoC) applications, but at the expense of increasing the complexity of the system or losing sensitivity. A new technology is being developed to build a $\mathrm{PoC}$ device based on fluorescence lifetime detection for molecular diagnosis with a sensitivity comparable to the bulky optical instruments and with a diagnosis time of a few seconds. Our PoC is a low-cost, simple, fast and easy to use general purpose platform, aimed at carrying out a fast diagnostics test through label detection of a variety of biomarkers. The system is designed to eliminate the optical requirements associated with traditional fluorescence lifetime instruments. With an array of ultra-sensitive detectors based on CMOS SAPD (single photo avalanche diode) technology along a custom microfluidic polydimethyl-siloxane (PDMS) cartridge on top of the sensor to insert the sample. The proximity of the sample and the SPAD sensor conjointly with the gate mode operation of the sensors, makes the use of lenses and optical filters unnecessary. The device is operated in Time Correlated Single Photon Counting (TCSPC), measuring the time of arrival of the photons after excitation of the fluorescence with a nanosecond laser diode. The sensor, which is extremely sensitive to light in the rage from $400 \mathrm{~nm}$ to $1000 \mathrm{~nm}$, and of ultra-high speed, works in gated mode, which makes it practically unaffected by the intrinsic noise. The system has been characterized with several concentrations of fluorescent quantum dots (Qdot ${ }^{\circledR} 605$ streptavidin conjugate from Life Technologies) as proof-of-concept for lifetimes of several nanoseconds. The lifetime of the quantum dots is $35 \mathrm{~ns}$, and is measured in only $15 \mathrm{~s}$.

Conflicts of Interest: The authors declare no conflict of interest.

(C) 2017 by the authors. Licensee MDPI, Basel, Switzerland. This article is an open access article distributed under the terms and conditions of the Creative Commons Attribution (CC BY) license (http://creativecommons.org/licenses/by/4.0/). 